\title{
Dissociation and Intrapelvic Entrapment of a Dual-mobility Polyethylene Component
}

\author{
Keith A. Fehring MD, Daniel J. Berry MD
}

Received: 30 January 2015/ Accepted: 28 May 2015/Published online: 19 June 2015

(C) The Association of Bone and Joint Surgeons (B) 2015

\begin{abstract}
Background Dual-mobility bearings have gained popularity in recent years as a proposed method of reducing the risk of dislocation after primary and revision hip arthroplasties. Intraprosthetic dislocation, defined as dissociation of the smaller femoral head from the larger outer polyethylene head, is a known complication of these designs. Intraprosthetic dislocation typically requires reoperation and revision as closed reduction of intraprosthetic dislocations of these components is not effective. The small femoral head typically remains inside the large diameter acetabular component during intraprosthetic dislocation, thus the diagnosis may be missed if femoral head eccentricity is not identified on radiographs. Intraprosthetic dislocation leads to a free polyethylene bearing which typically stays in the joint space, however in theory, migration of the bearing is possible.
\end{abstract}

One of the authors certifies that he (DJB), or a member of his or her immediate family, has or may receive payments or benefits, during the study period, an amount of USD (USD 100,001-USD 1,000,000 from Depuy Orthopaedics, Warsaw, IN, USA).

All ICMJE Conflict of Interest Forms for authors and Clinical Orthopaedics and Related Research ${ }^{\mathbb{B}}$ editors and board members are on file with the publication and can be viewed on request.

Clinical Orthopaedics and Related Research ${ }^{\circledR}$ neither advocates nor endorses the use of any treatment, drug, or device. Readers are encouraged to always seek additional information, including FDAapproval status, of any drug or device prior to clinical use.

Each author certifies that his or her institution approved or waived approval for the reporting of this case and that all investigations were conducted in conformity with ethical principles of research.

K. A. Fehring, D. J. Berry $(\square)$

Department of Orthopedic Surgery, Mayo Clinic, 200 First Street

SW, Rochester, MN 55905, USA

e-mail: Berry.Daniel@mayo.edu
Case Description We report a case of polyethylene bearing disengagement and intrapelvic entrapment after anterior dislocation of a hip with a modern dual-mobility bearing. The diagnosis of polyethylene component migration to an intrapelvic location was not made until surgery. Isolated acetabular revision of the dual-mobility bearing was performed to correct the instability in this patient. The entrapped polyethylene head was left in its intrapelvic position as retrieval was thought to be more morbid than retention of the component.

Literature Review Intraprosthetic dislocation, with disassociation of the polyethylene bearing from the femoral head, is a known complication of dual-mobility designs. Reports of intrapelvic entrapment of trial femoral heads of conventional hip arthroplasties have been described extensively in the literature. To our knowledge, intrapelvic migration and entrapment of a polyethylene bearing after dislocation of a hip with a dual-mobility implant has not been reported.

Clinical Relevance We discuss an implant-specific complication that has not been reported, and surgeons should be aware that this unique complication can occur. Surgeons should recognize the eccentricity of the femoral head on postreduction radiographs so an intraprosthetic dislocation is not missed. Furthermore, surgeons should be aware that intraprosthetic dislocation carries a risk of intrapelvic migration of the polyethylene component which is not visible on plain radiographs.

\section{Introduction}

The concept of dual-mobility hip articulations was introduced in 1974 by Bousquet in an effort to improve THA joint stability $[7,14,15]$. The use of dual-mobility 
components in primary and revision hip arthroplasties has increased during the last several years owing to design changes, recent approval for use in the United States, and surgeons' enthusiasm for bearings that may provide lower dislocation rates [1]. A dual-mobility bearing consists of two articulating surfaces. A large polyethylene head (bearing) articulates with a polished metal inside surface of a one-piece acetabular component or a polished metal liner of a modular acetabular component, and a smaller femoral head articulates with the inside of the larger polyethylene head. The smaller femoral head-to-polyethylene articulation typically is constrained and is assembled before implant insertion with a specialized instrument. This construct provides a large effective head diameter that increases ROM free of hard impingement of the femoral neck against the metal acetabular shell and increases jump distance, both of which theoretically decrease the risk of dislocation [6, 10]. The long-term use of dual-mobility designs in Europe has provided data regarding survivorship and complications associated with these implants [9, 10, 14, 15].

Intraprosthetic dislocation is a well-known complication specific to dual-mobility designs $[1,11,12,14,15]$. Intraprosthetic dislocation is defined as dissociation of the small-diameter femoral head from the larger outer polyethylene head in a dual-mobility bearing. In most cases, the smaller metal head remains in the metal acetabular component, therefore the term intraprosthetic dislocation. Philippot et al. [15] reported on the largest cohort of patients and has devised a classification system for intraprosthetic dislocation. Many of these dislocations occurred at mid-term followup because of repetitive soft impingement of the prosthetic femoral neck against the constrained polyethylene insert leading to breakdown of the constraining mechanism [9, 15].

To our knowledge, we present the first report of acute dissociation and intrapelvic migration and entrapment of the polyethylene component of a dual-mobility implant. As these implants increase in popularity, surgeons should be aware of this unique complication, understand keys to diagnosis of an intraprosthetic dislocation, recognize migration of the free polyethylene bearing is possible, and realize the clinical dilemma involved with retrieval versus retention of an entrapped intrapelvic component.

\section{Case Report}

A 59-year-old woman was referred to our institution for recurrent anterior hip dislocation 5 months after revision THA that was performed for posterior instability using a dual-mobility bearing implanted through a posterior approach at an outside institution. Four years before that revision, she had undergone a primary THA and had five episodes of posterior hip dislocation during the ensuing 4 years. The patient's BMI was $30.15 \mathrm{~kg} / \mathrm{m}^{2}$. The components at initial revision THA included a $54-\mathrm{mm}$ press-fit Tritanium $^{\circledR}$ acetabular component (Stryker ${ }^{\circledR}$, Mahwah, NJ, USA), a 54- × 42-mm metallic liner, a 42- $\times 28-\mathrm{mm}$ highly crosslinked polyethylene component (Modular Dual Mobility $\mathrm{X} 3{ }^{\circledR}$; Stryker $\left.^{\circledR}\right)$, and a $28-\mathrm{mm}+8.5-\mathrm{mm}$ ARTICUL/EZE ${ }^{\circledR}$ cobalt-chromium femoral head (DePuy, Warsaw, IN, USA). She had a retained well-fixed Anatomic Medullary Locking short-stature femoral stem (DePuy) that was in place from the primary THA.

Postoperative radiographs confirmed the femoral head was concentrically reduced in the acetabulum after the revision operation. After her revision arthroplasty, the hip dislocated five times anteriorly and the patient was treated with closed reduction after each occurrence. Each closed reduction at outside institutions was thought to have been successful. AP (Fig. 1A) and lateral (Fig. 1B) radiographs after the patient's first two dislocations were available for retrospective review; however, the concentricity of her femoral head was difficult to confirm because of the poor quality of the radiographs, soft tissue shadows, and density of the metal insert of the acetabular component. After her first closed reduction the patient began to notice an audible clunking in the hip with motion. Radiographs taken during her initial consultation at our institution showed an eccentrically located femoral head in the acetabulum (Fig. 2). An intraprosthetic dislocation was thought to be present and the patient was taken to the operating room for planned acetabular component revision.

At the time of revision arthroplasty, the metallic femoral head was found to be articulating directly with the metallic dual-mobility liner. On exploration of the left hip, the polyethylene component could not be located anywhere in the hip. We surmised the polyethylene component was located in an intrapelvic position, but by reaching around the pelvic brim, we could not palpate or reach it definitively, and we did not feel it could be safely retrieved without a separate incision and exploratory surgery. A decision was made to complete the revision arthroplasty without attempted retrieval of the polyethylene component.

The acetabular component was revised to a less abducted and slightly less anteverted position to improve stability (Fig. 3). The components placed at revision were a 60-mm press-fit Tritanium $^{\circledR}$ acetabular component, a 60- $\times$ 46-mm metallic liner, a 46- $\times$ 28-mm highly crosslinked polyethylene component (Modular Dual Mobility X3 ${ }^{\circledR}$; Stryker ${ }^{\circledR}$ ), and a $28 \mathrm{~mm}+8.5$-mm femoral head (DePuy). The femoral stem was found to be stable and was retained.

Postoperatively, CT of the pelvis confirmed intrapelvic location of the polyethylene component medial and superior to the anterior wall of the acetabulum (Fig. 4). No 

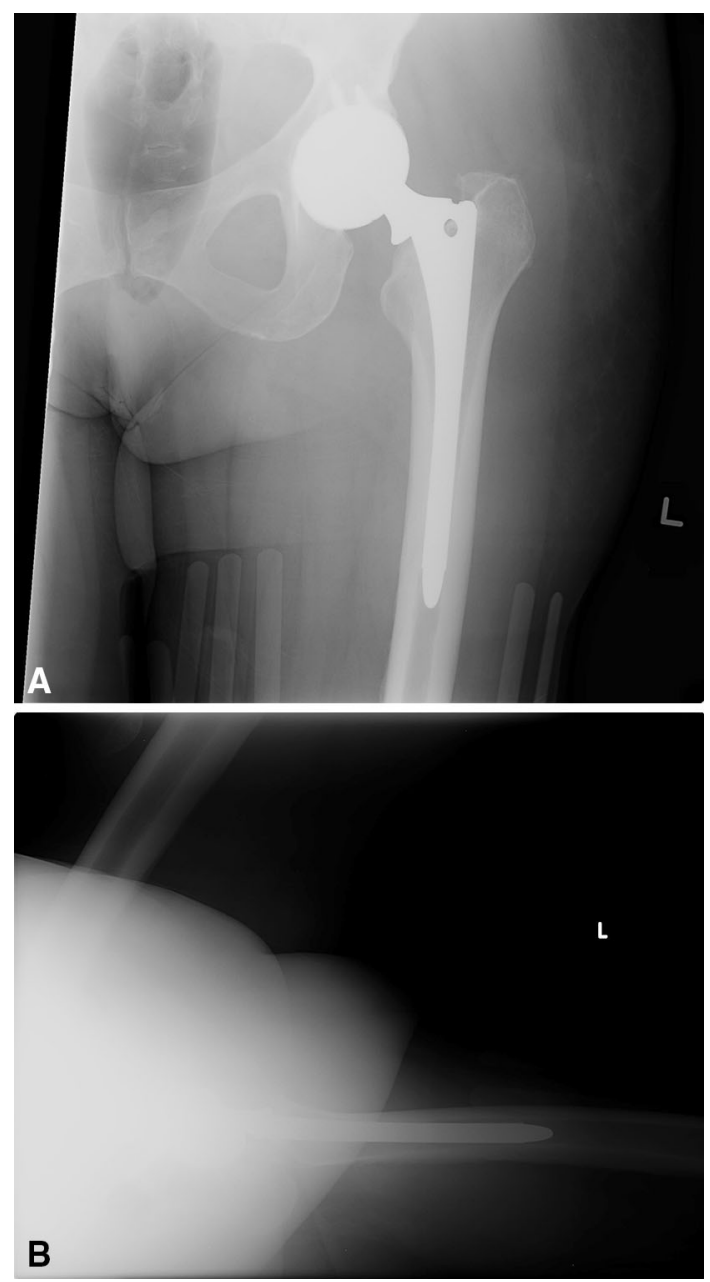

Fig. 1A-B Postreduction (A) AP and (B) lateral radiographs show that eccentricity of the femoral head was difficult to detect because of the soft tissue shadows and density of the metallic insert of the acetabular component.

compression of any intrapelvic vital structures was observed. A discussion was held with the patient concerning risks and potential benefits of a separate procedure with a retroperitoneal approach to retrieve the polyethylene insert. A joint decision was made not to retrieve the component, with the understanding that retrieval could be performed in the future should the intrapelvic component cause symptoms. At 3 months followup, the patient is doing well clinically. At this time, we do not plan to perform serial CT scans unless symptoms should arise.

\section{Discussion}

Enthusiasm for dual-mobility constructs in primary and revision THAs has increased in recent years owing to the potential for increased hip stability. A known complication
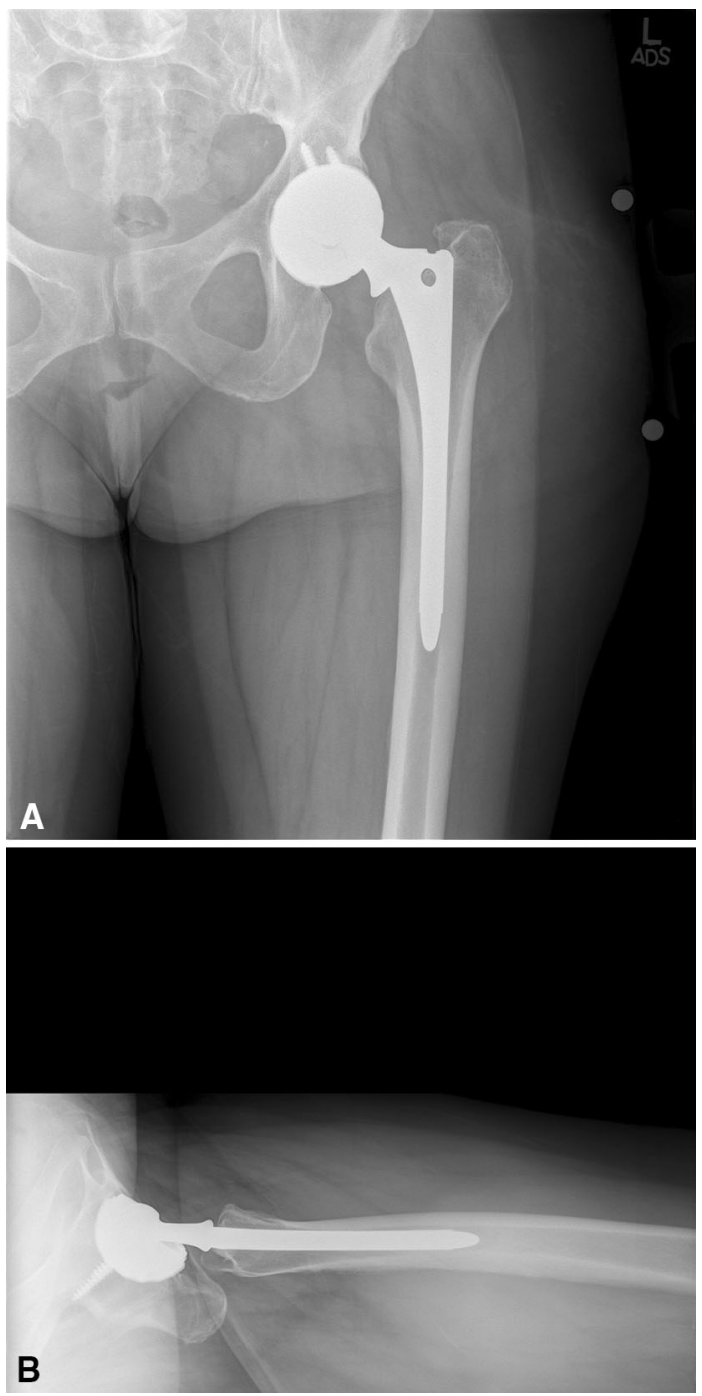

Fig. 2A-B (A) AP and (B) lateral radiographs show the eccentric position of the femoral head in the acetabulum.

of this category of implants is intraprosthetic dislocation (with disassociation of the larger polyethylene head from the smaller femoral head) with a long-term incidence reported at $2 \%$ to $4 \%$ [15]. In THAs using conventional components and modular trial implants, intrapelvic migration along the path of the psoas sheath of trial and final modular prosthetic femoral heads has been reported [3-5, 8, 13, 16, 17]. Several case reports have described intraprosthetic dislocations in which, at the time of revision, the polyethylene component was in the gluteal musculature or the hip capsule [1, 12, 15]. To our knowledge, this is the first report of intrapelvic migration and entrapment of a dual-mobility polyethylene component associated with dislocation of a dual-mobility bearing.

Surgeons should be aware of three main learning points associated with this complication: (1) The diagnosis of an intraprosthetic dislocation may be missed if radiographs 
are not high-quality AP and lateral views of the hip. The key to diagnosis is eccentricity of the femoral head in the acetabulum. (2) Once intraprosthetic dislocation has occurred, the free polyethylene bearing may migrate. (3) If intrapelvic migration of the component occurs, it makes for difficult decisions between the surgeon and patient regarding whether retrieval or retention is the best management. This diagnosis can be difficult because of radiolucency of the polyethylene component which may be difficult or impossible to identify on a standard radiograph. Even retrospectively, we were unable to see the shadow of the polyethylene component to identify its location on postreduction radiographs.

Surgeons should be aware of the potential for migration of the polyethylene bearing when performing revision surgery on a patient with apparent intraprosthetic dislocation of a

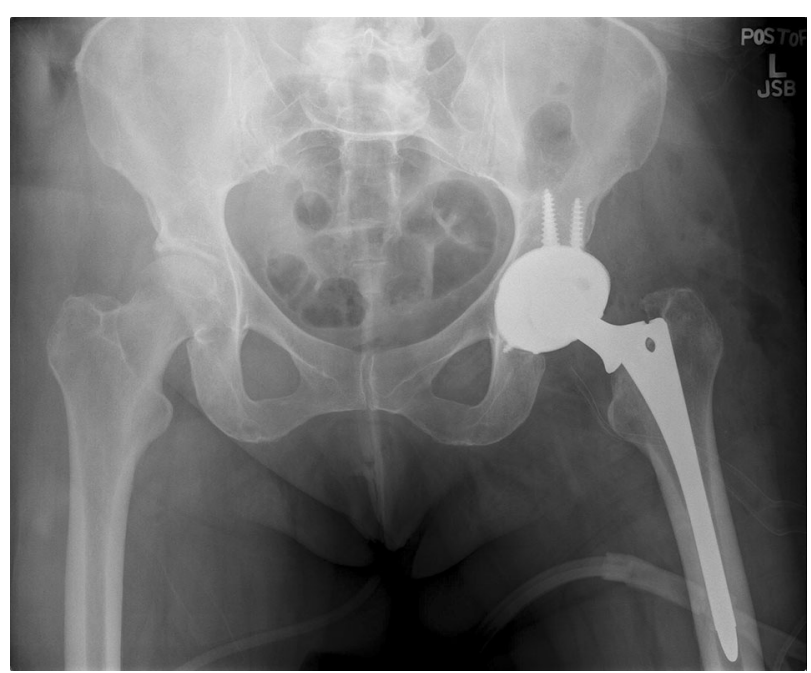

Fig. 3 An AP radiograph of the pelvis after revision THA is shown. dual-mobility construct. When intrapelvic migration of this component has occurred, a clinical dilemma exists regarding retrieval versus retention of the component. Patients understandably will be concerned by the idea of leaving the component in place, however education regarding the potential complications of retrieval including hernia, neurovascular injury, or urologic injury should be discussed. Retrieval of trial femoral heads entrapped in the pelvis may be performed through a separate surgical approach at the time of surgery $[3,5,13]$. One study regarding leaving trial femoral heads that have become entrapped intrapelvically did not identify adverse effects, and patients have remained asymptomatic when such implants have been left in an intrapelvic position [2]. Whether the morbidity of proceeding with a separate incision to retrieve an entrapped intrapelvic component is justified remains debatable. With our patient, because the retained component is a sterile, rounded, smooth polyethylene that does not appear to be compressing vital structures, a decision was made in consultation with the patient not to remove the implant.

Late polyethylene wear has been postulated as a potential mechanism of intraprosthetic dislocation in dualmobility designs [15]. Our patient sustained a dislocation early during her postoperative course making wear an unlikely cause of the disassociation of the smaller head from the polyethylene component. Retrospective review of serial radiographs in this case from outside institutions does not suggest any of the anterior hip dislocations were intrapelvic. Because the polyethylene component cannot be seen on radiographs, we are uncertain whether the polyethylene component was still attached to the femoral head at the time of the first anterior dislocation. One could postulate there was disengagement of the polyethylene component from the femoral head by disruption of the constrained polyethylene interface either at the time of hip
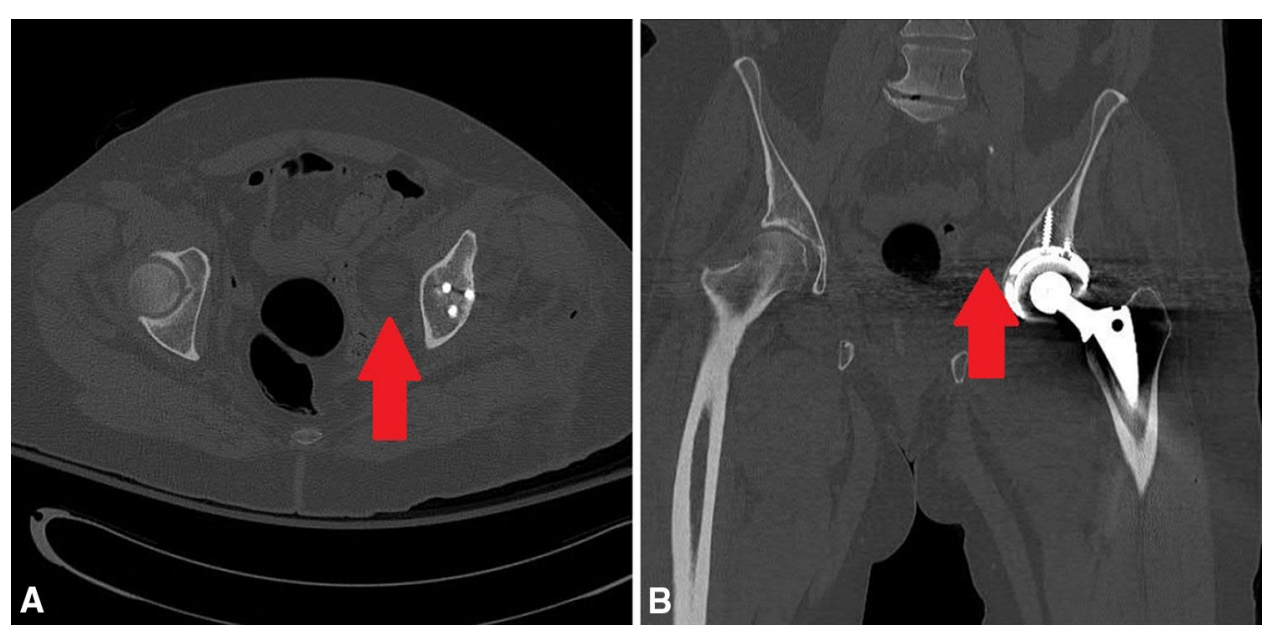

Fig. 4A-B (A) Axial and (B) coronal CT scans confirmed the intrapelvic location (arrow) of the polyethylene component. 
dislocation or during reduction of the hip. We theorize that the disassociated polyethylene component was pushed intrapelvically along the psoas sheath, either during one of the patient's closed hip reductions or during the course of normal hip motion. In our patient, the Stryker dualmobility acetabular system was used with a DePuy femoral head, which is considered an off-label use. However, to our knowledge, there are no major differences between Stryker ${ }^{\circledR}$ and DePuy cobalt-chrome 28-mm femoral heads.

Surgeons should be aware of intraprosthetic dislocation with dual-mobility articulations and the possibility that the polyethylene component could be entrapped in the pelvis after dislocation. Identification of eccentricity of the femoral head in the acetabular component after dislocation is essential to avoid missing the diagnosis of intraprosthetic dislocation in this setting. The possibility that the polyethylene component may not be retrievable may be discussed with the patient before surgery or preoperative cross-sectional imaging may be performed to definitively identify the location of the component before revision surgery.

\section{References}

1. Banzhof JA, Robbins CE, van der Ven A, Talmo CT, Bono JV. Femoral head dislodgement complicating use of a dual mobility prosthesis for recurrent instability. J Arthroplasty. 2013;28:543. e1-3.

2. Batouk O, Gilbart M, Jain R. Intraoperative dislocation of the trial femoral head into the pelvis during total hip arthroplasty: a case report. J Bone Joint Surg Am. 2001;83:1549-1551.

3. Callaghan JJ, McAndrew C, Boese CK, Forest E. Intrapelvic migration of the trial femoral head during total hip arthroplasty: is retrieval necessary? A report of four cases. Iowa Orthop J. 2006;26:60-62.

4. Chu CM, Wang SJ, Lin LC. Dissociation of modular total hip arthroplasty at the femoral head-neck interface after loosening of the acetabular shell following hip dislocation. J Arthroplasty. 2001;16:806-809.

5. Citak M, Klatte TO, Zahar A, Day K, Kendoff D, Gehrke T, Dörner A, Gebauer M. Intrapelvic dislocation of a femoral trial head during primary total hip arthroplasty requiring laparotomy for retrieval. Open Orthop J. 2013;7:169-171.

6. De Martino I, Triantafyllopoulos GK, Sculco PK, Sculco TP. Dual mobility cups in total hip arthroplasty. World J Orthop. 2014;5:180-187.

7. Farizon F, de Lavison R, Azoulai JJ, Bousquet G. Results with a cementless alumina-coated cup with dual mobility: a twelve-year follow-up study. Int Orthop. 1998;22:219-224.

8. Kouzelis A, Georgiou CS, Megas P. Dissociation of modular total hip arthroplasty at the neck-stem interface without dislocation. J Orthop Traumatol. 2012;13:221-224.

9. Lachiewicz PF, Watters TS. The use of dual-mobility components in total hip arthroplasty. J Am Acad Orthop Surg. 2012;20: $481-486$

10. McArthur BA, Nam D, Cross MB, Westrich GH, Sculco TP. Dual-mobility acetabular components in total hip arthroplasty. Am J Orthop (Belle Mead NJ). 2013;42:473-478.

11. Mohammed R, Cnudde P. Severe metallosis owing to intraprosthetic dislocation in a failed dual-mobility cup primary total hip arthroplasty. J Arthroplasty. 2012;27:493.e1-3.

12. Odland AN, Sierra RJ. Intraprosthetic dislocation of a contemporary dual-mobility design used during conversion THA. Orthopedics. 2014;37:e1124-1128.

13. Ozkan K, Ugutmen E, Altintas F, Eren A, Mahirogullari M. Intraoperative dislocation of the prosthetic femoral head into the pelvis during total hip arthroplasty. Acta Orthop Belg. 2008; 74:553-555.

14. Philippot R, Adam P, Farizon F, Fessy MH, Bousquet G. [Survival of cementless dual mobility sockets: ten-year follow-up][in French]. Rev Chir Orthop Reparatrice Appar Mot. 2006;92:326331.

15. Philippot R, Boyer B, Farizon F. Intraprosthetic dislocation: a specific complication of the dual-mobility system. Clin Orthop Relat Res. 2013;471:965-970.

16. Talmo CT, Sharp KG, Malinowska M, Bono JV, Ward DM, LaReau J. Spontaneous modular femoral head dissociation complicating total hip arthroplasty. Orthopedics. 2014;37:e592595.

17. Ziv YB, Backstein D, Safir O, Kosashvili Y. Intraoperative dislocation of a trial femoral head into the pelvis during total hip arthroplasty. Can J Surg. 2008;51:E73-74. 